\title{
An Uncertainty-Aware Online Planning Algorithm for the Sustainable Electrification of Festivals
}

\author{
Iliana Pappi, Tim Baarslag, Michael Kaisers \\ Centrum Wiskunde \& Informatica \\ The Netherlands \\ iliana.n.pappi@gmail.com; \{t.baarslag, michael.kaisers\}@cwi.nl
}

\author{
Nikolaos G. Paterakis \\ Eindhoven University of Technology \\ The Netherlands \\ n.paterakis@tue.nl
}

\begin{abstract}
Energy efficient festival electrification can be viewed as a middle-consumption problem, standing between smart household applications and larger commercial consumers. The optimal deployment of different resources, such as local renewable energy production (RES), diesel generators (DG) and energy storage systems (ESS) may bring about significant financial gain for the organizer, and is usually framed as an off-grid or limited grid-connection problem. This makes online planning particularly challenging due to the uncertainty related to the RES production, constraints regarding the operation of the diesel generators and limitations of the grid connection. In this paper a new online planning algorithm based on two-stage stochastic programming is proposed in order to address the aforementioned challenges and provide minimal-cost, uninterrupted, and sustainable electrification of festivals under dynamically priced grid energy. Data based on real festival events are used in order to illustrate the effectiveness of the proposed methodology.

Keywords-energy storage, festival electrification, online planning, renewable energy, stochastic programming.
\end{abstract}

\section{INTRODUCTION}

The exploitation of renewable energy sources (RES) is a topic that has been studied extensively and from various perspectives in the last decades. The integration of RES at the level of the bulk electrictiy system is challenging due to the uncertainty and variability inherent to their generation, which in turn can increase the need in procuring pricey ancillary services (e.g., regulation, reserves, etc.) to ensure system stability and reliability [1]. Apart from grid-scale applications, RES can be also used for the electrification of remote areas and other standalone applications. Moreover, RES can be installed behind-the-meter and combined with other resources in order to reduce the dependency of a consumer on the grid connection.

The electrification of outdoor festival events is an example of the last category of applications that is challenging both in terms of selecting the type and size of resources to be used and its real-time operation in order to guarantee minimal-cost and uninterrupted supply of the demand. An energy management tool is expected to make schedules ahead of time, while the generation of the utilized RES production is still uncertain. However, this may lead to sub-optimal or even infeasible solutions, if poor forecasts are used both for the day- or hour-ahead planning. Several solutions have been suggested to this problem, such as the use of energy storage systems (ESS) and demand response (DR) in order to manage both the unpredictability of RES generation and follow more closely the real-time availability of RES generation

Several literature studies are devoted to addressing these issues at a microgrid or a nanogrid scale. However, to the best knowledge of the authors, the energy management problem of festival electrification has not been studied yet in the relevant literature. Madaeni et al. [2] proposed a stochastic optimization framework to investigate the relative benefits of DR and real-time pricing (RTP) in reducing the impact of RES on system costs. Niknam et al. [3] proposed a multiobjective cost-emission minimization stochastic algorithm for microgrids. Moreover, Cardoso et al. [4] developed a microgrid reliability model handling uncertainty related to fuel cell module production in the day-ahead battery scheduling. On the contrary, a real-time DR management model based on stochastic and robust optimization was proposed in [5]. However, this model is restricted to residential appliances. Finally, a deterministic rolling-horizon decision-making tool tailored to residential end-users was proposed in [6]. This study indicated how updated information on the uncertain parameters can be exploited in improving costs by repeatedly executing the energy management algorithm. Based on the evidence provided by the aforementioned studies, online planning algorithms constitute an effective approach to managing uncertainty by closely following the real-time conditions.

Despite the rich body of literature devoted to the development of energy management systems in various setups, to the best knowledge of the Authors, the electrification of festivals is an energy management problem of a medium consumer that has not been studied in the relevant literature. The contribution of this paper is twofold:

- The principled treatment of the energy management problem of a special type of electrical energy consumer that typically relies on simple rule-of-thumb approaches.

- The development of an online planning tool based on twostage stochastic programming for the energy management of festival events.

In this study, the underlying optimization model is cast as a mixed-integer linear programming (MILP) optimization problem in order to exploit the computational efficiency of state-of-the-art solvers. Accurate parametric forecasts are difficult to attain and often do not easily combine with available 
forecasting systems. For this reason, a non-parametric set of scenarios is employed in order to quantify uncertainty in the generation of photovoltaic (PV) systems and clustering is used to compile a smaller representative scenario centroid set with associated probabilities. Furthermore, the execution of the planning algorithm is repeated within the duration of the festival event, incorporating new information on the realization of the uncertain variables and improving the quantification of uncertainty by updating the set of the scenarios that are being considered. The festival organizer considers the so-called hereand-now decisions in order to optimally procure energy from its own behind-the-meter assets and the grid connection, with the prupose of economically covering the load demand for the time intervals between two executions of the algorithm. One significant advantage is that, unlike deterministic or simple rule-of-thumb approaches, feasibility is guaranteed for all the plausible realizations of uncertainty by reserving adequate capacity from other controllable resources.

The remainder of this paper is organized as follows: in Section II the proposed optimization model is developed and a benchmark approach is established. A case study is presented in Section III. Finally, conclusions are drawn in Section IV.

\section{Methodology}

\section{A. Model}

The objective function stands for the minimization of the expected cost (EC) and is represented by (1). It is assumed that the festival organizer buys energy from the grid at an hourly-varying price.

$$
\begin{aligned}
E C & =\sum_{t}\left(c_{l t}^{\text {diesel }} \cdot \gamma \cdot P_{t}^{D G}+c_{t}^{\text {grid }} \cdot P_{t}^{g r i d}\right) \cdot \Delta T \\
& +\sum_{s} \pi(s) \cdot \sum_{t}\left[c_{l t}^{\text {diesel }} \cdot \gamma \cdot\left(p_{s, t}^{D G}-P_{t}^{D G}\right)\right. \\
& +c_{t}^{\text {grid }} \cdot\left(r_{s, t}^{\text {grid,u }}-r_{s, t}^{\text {grid, }}\right) \\
& \left.+M_{\text {spill }} \cdot s p_{s, t}^{P V}+M_{\text {shed }} \cdot l s_{s, t}^{P V}\right] \cdot \Delta T
\end{aligned}
$$

The objective function consists of two parts. The first part involves decision variables that are not dependent on any specific realization of uncertainty and stand for here-and-now decisions that are made before the realization of uncertainty. The second part involves variables that depend on a specific scenario realization and account for the cost of corrective actions. Thus, they represent wait-and-see decisions [7]. The notation used in this work is described in Table I.

The first-stage constraints of the problem, involving only variables that are not dependent on any specific scenario realization, are described by (2)-(14). The power-balance is expressed by (2). Grid connection limitations are taken into account by (3) and (4). Constraints on the planned production of the diesel generator (DG), as well as the corresponding up and down reserve limits, are enforced by (5) and (6). Furthermore, (7) considers limitations on the maximum time that the DG can be operated, typically imposed by renting

\begin{tabular}{|c|c|}
\hline & Sets \\
\hline \multirow{4}{*}{$\begin{array}{l}h(H) \\
t(T) \\
s(\Omega) \\
\end{array}$} & index (set) of optimization repetition intervals \\
\hline & index (set) of time periods \\
\hline & index (set) of scenarios \\
\hline & Parameters \\
\hline$c_{l t}^{\text {diesel }}$ & price of diesel $(€ / l t)$ \\
\hline$c_{t}^{g r i d}$ & grid energy price in period $t(€ / k W h)$ \\
\hline$\gamma$ & fuel to power conversion constant $(l t / k W h)$ \\
\hline$N_{\text {grid }}$ & restriction to power drawn from the grid \\
\hline$M_{\text {spill }}$ & RES production spillage penalty $(€ / k W h)$ \\
\hline$M_{\text {shed }}$ & load shedding penalty $(€ / k W h)$ \\
\hline$P_{\min }^{D G} / P_{\max }^{D G}$ & $\mathrm{~min} / \mathrm{max}$ diesel generator (DG) output $(\mathrm{kW})$ \\
\hline$T_{D G, o n}^{m a x}$ max & maximum time intervals a DG can be used in $T$ \\
\hline & duration of time intervals $(h)$ \\
\hline$P_{E S S}^{\text {rated }}$ & energy storage system (ESS) rated power $(k W)$ \\
\hline$S O E^{\min } / S O E^{\max }$ & ESS state-of-energy limits $(k W h)$ \\
\hline$S O E^{i n i}$ & initial state-of-energy of ESS $(k W h)$ \\
\hline$C E_{E S S} / D E_{E S S}$ & charging /discharging efficiency of ESS \\
\hline & load in period $t(k W)$ \\
\hline \multirow[t]{2}{*}{$\begin{array}{l}P V_{t, s} \\
\pi(s)\end{array}$} & $\begin{array}{l}\text { PV production in period } t \text { and scenario } s(k W) \\
\text { probability of scenario } s\end{array}$ \\
\hline & Continuous variables \\
\hline$E C$ & expected cost $(€)$ \\
\hline$P_{t}^{P V}$ & PV power in period $t(k W)$ \\
\hline$P_{t}^{D G}$ & DG power in period $t(k W)$ \\
\hline$R_{t}^{D G, u} / R_{t}^{D G, d}$ & scheduled DG up/down reserve in period $t(k W)$ \\
\hline$P_{t}^{g r i d}$ & grid power in period $t(k W)$ \\
\hline$R_{t}^{g r i d, u} / R_{t}^{g r i d, d}$ & scheduled grid up/down reserve in period $t(k W)$ \\
\hline$P_{t}^{c h} / P_{t}^{\text {dis }}$ & ESS charging/discharging power in period $t(k W)$ \\
\hline$S O E_{t}$ & ESS state-of-energy in period $t(k W h)$ \\
\hline$p_{s, t}^{D G}$ & DG power in period $t$ and scenario $s(k W)$ \\
\hline$r_{s, t}^{\bar{D} G, u} / r_{s, t}^{D G, d}$ & $\begin{array}{l}\text { used DG up/down reserve in period } t \\
\text { and scenario } s(k W)\end{array}$ \\
\hline$p_{s, t}^{g r i d}$ & grid power in period $t$ and scenario $s(k W)$ \\
\hline$r_{s, t}^{\text {grid }, u} / r_{s, t}^{\text {grid }, d}$ & $\begin{array}{l}\text { used up/down grid reserve in period } t \\
\text { and scenario } s(k W)\end{array}$ \\
\hline$s p_{s, t}^{P V}$ & $\mathrm{PV}$ spillage in period $t$ and scenario $s(k W)$ \\
\hline$l s_{s, t}^{P}+V$ & load shedding in period $t$ and scenario $s(k W)$ \\
\hline$p_{s, t}^{c h} / p_{s, t}^{d i s}$ & $\begin{array}{l}\text { charging/discharging power of ESS in period } t \\
\text { and scenario } s(k W)\end{array}$ \\
\hline $\operatorname{soe}_{s, t}$ & $\begin{array}{l}\text { ESS state-of-energy in period } t \\
\text { and scenario } s(k W h)\end{array}$ \\
\hline \multirow[t]{2}{*}{$\Delta P_{s, t}^{c h} / \Delta P_{s, t}^{d i s}$} & $\begin{array}{l}\text { change in charging/discharging power } \\
\text { in period } t \text { and scenario } s(k W)\end{array}$ \\
\hline & Binary variables \\
\hline$u_{t}^{D G}$ & 1 if DG is online in period $t$ \\
\hline$u_{t}^{c h}$ & 1 if ESS is charging in period $t$ \\
\hline$u_{s, t}^{c h 2}$ & $\begin{array}{l}1 \text { if ESS is charging in period } t \\
\text { and scenario } s\end{array}$ \\
\hline$u_{s, t}^{D G, r e s}$ & $\begin{array}{l}1 \text { if upward reserve from DG is used in period } t \\
\text { and scenario } s\end{array}$ \\
\hline$u_{s, t}^{d g 2}$ & 1 if DG is online in period $t$ and scenario $s$ \\
\hline$u_{s, t}^{g r i d, r e s}$ & $\begin{array}{l}1 \text { if upward reserve from the grid is used in } \\
\text { period } t \text { and scenario } s\end{array}$ \\
\hline
\end{tabular}
such equipment for specific times. Finally, the planned PV
TABLE I

NOMENCLATURE

power usage is constrained by the maximum production in the considered scenarios (8).

$$
\begin{gathered}
P_{t}^{\text {grid }}+P_{t}^{P V}+P_{t}^{D G}+P_{t}^{\text {dis }}=L_{t}+P_{t}^{c h} \\
P_{t}^{\text {grid }}+R_{t}^{\text {grid }, u} \leq N_{\text {grid }} \cdot \max _{t} L_{t} \\
R_{t}^{\text {grid, }, d} \leq P_{t}^{\text {grid }}
\end{gathered}
$$




$$
\begin{gathered}
P_{t}^{D G}+R_{t}^{D G, u} \leq P_{\max }^{D G} \cdot u_{t}^{D G} \\
P_{t}^{D G}-R_{t}^{D G, d} \geq P_{\min }^{D G} \cdot u_{t}^{D G} \\
\sum_{t} u_{t}^{D G} \leq T_{D G, \text { on }}^{\max } \\
P_{t}^{P V} \leq \max _{s} P V_{t, s}
\end{gathered}
$$

The rest of the first-stage constraints (9)-(14) describe the battery-based ESS constraints.

$$
\begin{gathered}
P_{t}^{c h} \leq P_{E S S}^{\text {rated }} \cdot u_{t}^{c h} \\
P_{t}^{d i s} \leq P_{E S S}^{r a t e d} \cdot\left(1-u_{t}^{c h}\right) \\
S O E_{t}=S O E_{t-1}+C E_{E S S} \cdot P_{t}^{c h} \cdot \Delta T \\
-D E_{E S S} \cdot P_{t}^{d i s} \cdot \Delta T \forall t>1 \\
S O E_{t}=S O E^{i n i}+C E_{E S S} \cdot P_{t}^{c h} \cdot \Delta T \\
-D E_{E S S} \cdot P_{t}^{d i s} \cdot \Delta T, t=1 \\
S O E_{t} \leq S O E^{\text {max }} \\
S O E_{t} \geq S O E^{\min }
\end{gathered}
$$

The second-stage constraints, that involve only variables that are dependent on specific scenario realizations, and the linking constraints, which involve both types of variables, are described by (15)-(39). The ESS constraints are expressed by (15)-(24). The differences in charging and discharging power between the first and the second stage are expressed by (15) and (17), while upper and lower bounds are established by (19) and (20) and by (16) and (18), respectively. Constraints (21) - (24) are the scenario dependent counterparts of (9)-(12).

$$
\begin{gathered}
p_{s, t}^{c h}=P_{t}^{c h}+\Delta P_{s, t}^{c h} \\
\Delta P_{s, t}^{c h} \geq-P_{t}^{c h} \\
p_{s, t}^{d i s}=P_{t}^{d i s}+\Delta P_{s, t}^{d i s} \\
\Delta P_{s, t}^{d i s} \geq-P_{t}^{d i s} \\
\Delta P_{s, t}^{c h} \leq P_{E S S}^{r a t e d}-P_{t}^{c h} \\
\Delta P_{s, t}^{d i s} \leq P_{E S S}^{r a t e d}-P_{t}^{d i s} \\
p_{s, t}^{c h} \leq P_{E S S}^{r a t e d} \cdot u_{s, t}^{c h 2} \\
p_{s, t}^{d i s} \leq P_{E S S}^{r a t e d} \cdot\left(1-u_{s, t}^{c h 2}\right) \\
\text { soe }_{s, t}=s o e_{s, t-1}+C E_{E S S} \cdot p_{s, t}^{c h} \cdot \Delta T \\
-D E_{E S S} \cdot p_{s, t}^{\text {dis }} \cdot \Delta T \forall t>1 \\
\text { soe }_{s, t}=S O E^{i n i}+C E_{E S S} \cdot p_{s, t}^{c h} \cdot \Delta T \\
-D E_{E S S} \cdot p_{s, t}^{\text {dis }} \cdot \Delta T, t=1
\end{gathered}
$$

The power balance in the second-stage is expressed by (25), while the limitations for scenario-dependent load shedding and available PV production spillage are expressed by (26) and (27), respectively.

$$
\begin{gathered}
P V_{t, s}-s p_{s, t}^{P V}+p_{s, t}^{D G}+p_{s, t}^{g r i d}+p_{s, t}^{d i s} \\
=L_{t}-l s_{s, t}^{P V}+p_{s, t}^{c h} \\
\quad s_{s, t}^{P V} \leq L_{t} \\
s p_{s, t}^{P V} \leq P V_{t, s}
\end{gathered}
$$

Restrictions regarding the energy procurement from the grid are described by the following set of equations. In (28)-(30) the up and down reserves that are deployed in the second stage are within the limits imposed by the first stage, showing the possibility of increasing or decreasing grid participation in a scenario realization. The maximum of up or down reserve is constrained by a limit imposed by the network maximum participation in covering the energy needs of the event over the planning horizon, as indicated by (31) and (32). The use of a binary variable prevents up and down reserves from having a nonzero value simultaneously.

$$
\begin{gathered}
p_{s, t}^{\text {grid }}=P_{t}^{g r i d}+r_{s, t}^{g r i d, u}-r_{s, t}^{g r i d, d} \\
r_{s, t}^{g r i d, u} \leq R_{t}^{g r i d, u} \\
r_{s, t}^{g r i d, d} \leq R_{t}^{g r i d, d} \\
r_{s, t}^{g r i d, u} \leq N_{g r i d} \cdot \max _{t} L_{t} \cdot u_{s, t}^{\text {grid,res }} \\
r_{s, t}^{g r i d, d} \leq N_{\text {grid }} \cdot \max _{t} L_{t} \cdot\left(1-u_{s, t}^{\text {grid,res }}\right)
\end{gathered}
$$

The last set of second-stage and linking constraints are related to the DG model and are expressed by (33)-(39), restricted again by the first-stage reserves.

$$
\begin{gathered}
p_{s, t}^{D G}=P_{t}^{D G}+r_{s, t}^{D G, u}-r_{s, t}^{D G, d} \\
r_{s, t}^{D G, u} \leq R_{t}^{D G, u} \\
r_{s, t}^{D G, d} \leq R_{t}^{D G, d} \\
r_{s, t}^{D G, u} \leq P_{\max }^{D G} \cdot u_{s, t}^{D G, r e s} \\
r_{s, t}^{D G, d} \leq P_{\max }^{D G} \cdot\left(1-u_{s, t}^{D G, r e s}\right) \\
p_{s, t}^{D G} \leq P_{\max }^{D G} \cdot u_{s, t}^{d g 2} \\
p_{s, t}^{D G} \geq P_{\min }^{D G} \cdot u_{s, t}^{d g 2}
\end{gathered}
$$

\section{B. Scenario generation and reduction}

The PV production scenario generation and reduction is based on machine learning techniques. Initially, a number of k-Nearest Neighboors Regression models are fit to different time spans of historical data in order to obtain an initial pool of equiprobable scenarios. Then, a scenario reduction technique based on the k-means clustering algorithm is used in order to obtain a reduced number of non-equiprobable scenarios that coincide with the cluster centroids. The probability $\pi(s)$ of each scenario is calculated based on the number of initially generated scenarios assigned to each cluster. 


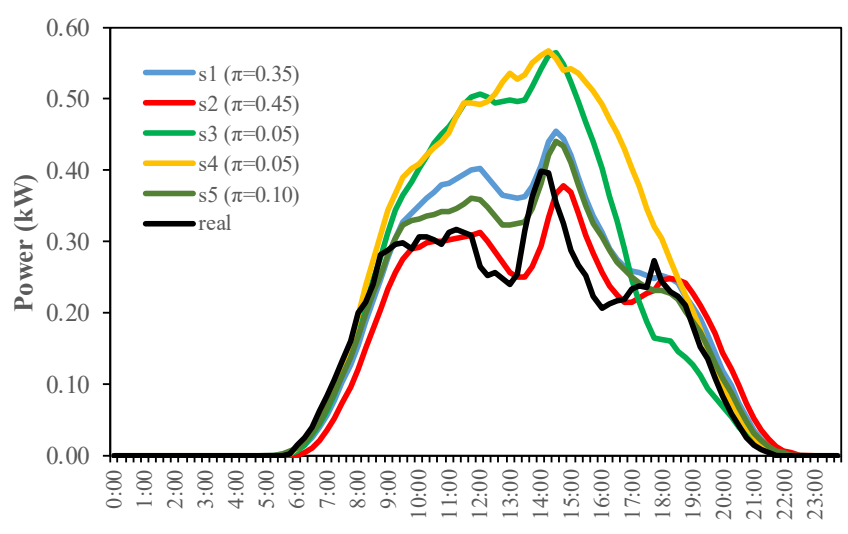

Fig. 1. Day-ahead PV production scenarios and their associated probabilities

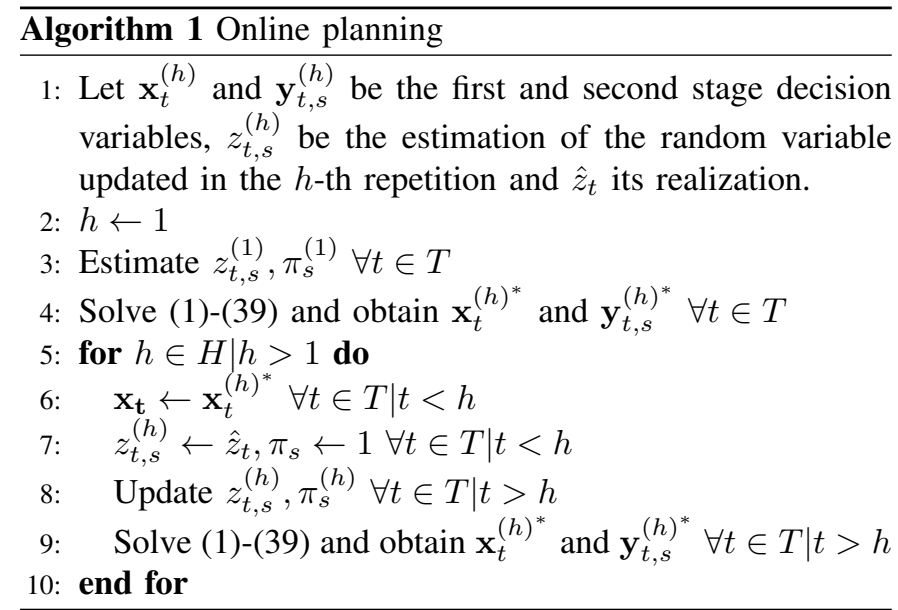

\section{Online planning}

The model described by (1)-(39) provides the optimal planning for $T$ future periods at the time it is executed. The solution of the optimization problem can be obtained again in the current time interval $h$ for the remaining time periods $t$ of the planning horizon. In this way, the estimation of the random variable that is associated with the production of the PV system can be improved in terms of updating the set of scenarios by repeating the scenario generation and reduction technique described in Section II-B, while incorporating newly available information. This procedure is described by Algorithm 1 .

\section{Evaluation}

If the scenarios and probabilities are accurate, our method computes (and in fact minimizes) expected costs that correspond to average actual costs. The introduction of a range of possible scenario realizations however, requires an evaluation method to test the feasibility of the algorithm and evaluate the fluctuation of the expected cost. Hence, to demonstrate the value of quantifying uncertainty in terms of using the online two-stage stochastic programming tool, a benchmark comparison with a deterministic approach of testing the model was established.
TABLE II

CASE STUdy Parameters

\begin{tabular}{ll||ll}
\hline Parameter & Value & Parameter & Value \\
\hline$c_{l t}^{\text {diesel }}$ & $1.21 € / l t$ & $\Delta T$ & 0.25 \\
$\gamma$ & $0.2166 \mathrm{lt} / \mathrm{kWh}$ & $P_{E S S}^{\text {rated }}$ & $60 \mathrm{~kW}$ \\
$N_{\text {grid }}$ & $0.2(20 \%)$ & $S O E^{\text {min } / \text { max }}$ & $0 / 100 \mathrm{kWh}$ \\
$P_{\min / \max }^{D}$ & $0 / 40 \mathrm{~kW}$ & $S O E^{\text {ini }}$ & $50 \mathrm{kWh}$ \\
$T_{D G \text { man }}^{\text {max }}$ & 36 intervals (8hrs) in $T$ & $C E / D E_{E S S}$ & $0.9 / 0.9$ \\
\hline
\end{tabular}

The model was executed for each one of the final set of scenarios created using the procedure described in Section II-B and the day-ahead results of the first-stage variables are stored. In other words a here-and-now decision is stored as a result of an optimization execution for a single scenario each time, as it would be the realized one in a deterministic approach. Following this, the model is re-executed for each one of the remaining scenarios with the first-stage variables fixed to the values that were determined for the realized scenario, while the imposed limit to grid connection is removed, and the secondstage variables are free to take a value in the second execution.

\section{CASe Study}

Our test involves a case study using data from real summer festival events in the Netherlands. Two months of historical PV production public datasets were used for scenario generation [8]. Fig. 1 depicts a reduced set of scenarios that are generated based on data available at the beginning of the horizon, together with the actual PV production. The assetrelated parameters of this case study are provided in Table II and are in line with those used by Dutch festival organizers. Hourly electricity prices of the Dutch day-ahead market were obtained from [9] for 12/7/2015. Based on field measurements the cost of operating the DG can be approximated as a linear function of fuel consumption. The power drawn from the grid is restricted to $20 \%$ of the maximum load. The penalization Mparameters both for load shedding and the spillage of available PV production were set to $1000 € / k W h$ in order to highlight the importance of uninterrupted power supply and sustainable electrification. The time granularity used is $15 \mathrm{~min}$. For the optimization model GAMS/CPLEX were used.

\section{A. Day-ahead Planning}

First, the proposed algorithm and the evaluation approach are executed at the beginning of the time horizon, providing the day-ahead planning. The expected cost is $29.85 €$, presented as the first execution of the online algorithm later in Table III. The day-ahead power analytics are shown in Fig. 2. The quantities that are illustrated correspond to the first-stage decision variables $P_{t}^{P V}, P_{t}^{D G}, P_{t}^{\text {grid }}, P_{t}^{c h, E S S}, P_{t}^{\text {dis, ESS }}$, as well as the load demand $L_{t}$. Note that the algorithm fully exploits the PV production, while despite being limited to $20 \%$ of the maximum load, power is drawn from the grid in every time interval due to the fact that it is less costly than the energy provided by the DG. Moreover, the ESS charging is mainly taking place during the time intervals in 


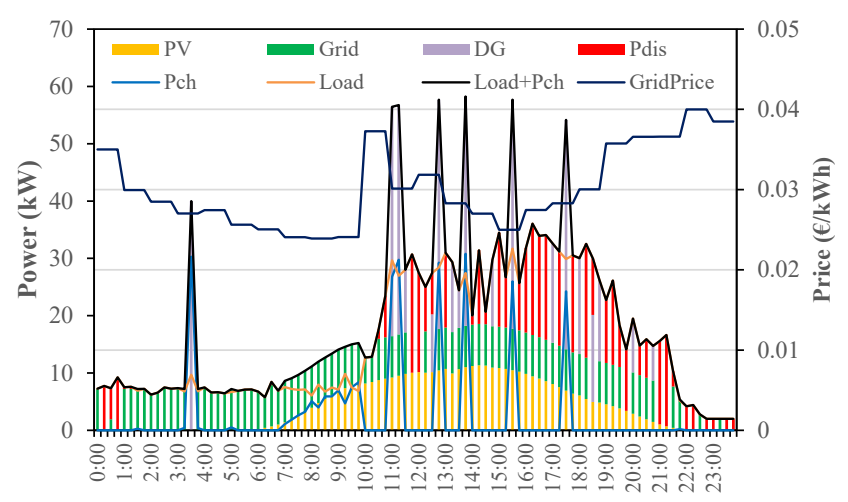

Fig. 2. Day-ahead power analytics

which the grid electricity price is relatively low. The ESS discharging is planned during intervals with high consumption and relatively high electricity prices. The DG is also used during high demand periods, and the load shedding is zero throughout the horizon.

The day-ahead evaluation as described in Section II-D proved to give feasible results for every scenario in the deterministic benchmark approach. The absolute differences between the one-scenario solution of the optimization model and the re-optimization of the second-stage for the remaining scenarios after fixing the first-stage variable values to the chosen scenario demonstrate an average absolute difference of $0.468 €$ and variance of $0.249 €$. The evaluation shows the feasibility and supports the solution quality, as the absolute differences in the expected cost are relatively low. The algorithm can offer a solution for all variables even if the input is a single scenario considered as the realized one and tested versus each of the remaining scenarios.

\section{B. Online Planning}

The online planning algorithm described in Section II-C is executed in every time interval $h$ (every $15 \mathrm{~min}$ ) for all the future time intervals $t>h$. The PV production scenarios are renewed for $t>h$ with newly available data of real PV production for the time intervals $t<h$. The past PV production with respect to the time interval $h$ in which the optimization is executed are fixed to the actual PV production values.

Furthermore, all the decision variables are fixed for the solutions of the previous time intervals $(t<h)$ and the $S O E_{t}^{E S S}$ value for the previous time interval $t=h-1$ updates the value of the $S O E_{E S S}^{i n i}$ of current time interval $h$. The estimation of the planning costs in key time intervals is presented in Table III. The feasibility of the planning solutions offered by the online planning tool is indicated by the fact that neither load shedding nor spillage of available PV production is noticed for the subsequent repetitions of the algorithm. The aforementioned results ensure that the uninterrupted electrification of a festival event is possible by using the proposed online planning tool, while the resulting cost approaches perfect knowledge of the uncertain PV production.

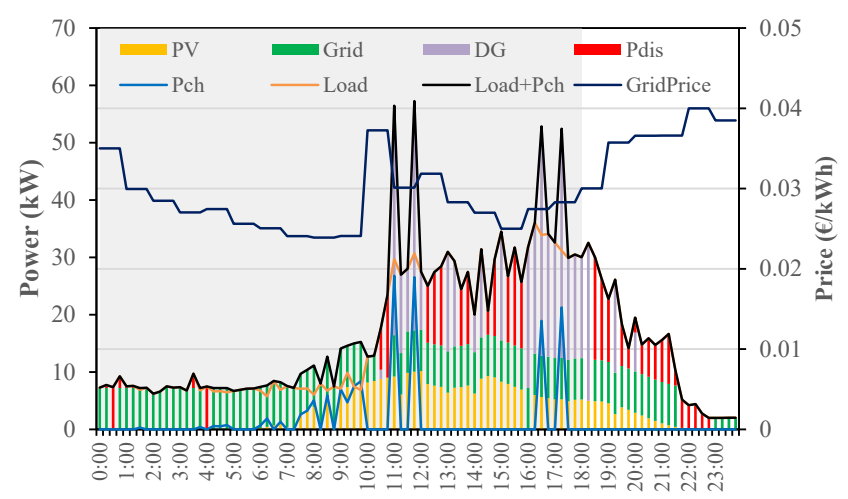

Fig. 3. Online planning power analytics $-6 \mathrm{pm}$.

TABLE III

OnLine Planning Cost Minimization

\begin{tabular}{lccccc}
\hline$h$ & day-ah.(12am) & $6 \mathrm{am}$ & $12 \mathrm{pm}$ & $6 \mathrm{pm}$ & end(11.45pm) \\
\hline$E C(€)$ & 29.85 & 29.85 & 30.9 & 31.36 & 32.09 \\
\hline
\end{tabular}

The power analytics for the optimization execution that takes place at $6 \mathrm{pm}$ are displayed in Fig. 3. The lightly shaded area before $t=72$ indicates time periods in which the decision variables are fixed to their realized values from previous executions of the algorithm and the PV production is set to its actual value. On top of previous fixed decisions the change on PV scenarios renewed by the new production data within the day affects the new planning. For example, the energy that is drawn by the ESS is increased in order to cover the consumption needs in the last intervals of the planning horizon. This result also reinforces the feasibility argument: there is sufficient energy left in the battery in combination with adequate capacity of resources even during the last intervals of the horizon.

\section{Conclusions}

In this study an uncertainty-aware online planning algorithm for the electrification of festival events based on two-stage stochastic mixed-integer linear programming was presented. The obtained results indicate that despite the increased complexity in comparison with simpler algorithms, the proposed algorithm is capable of offering a planning solution that guarantees the uninterrupted electrification of festival events at a cost that approaches the optimal cost of a deterministic optimization that assumes perfect knowledge of the uncertain photovoltaic production. This is achieved by adapting newly available information on the realization of the uncertain variable that allows for improvements in the the quantification of uncertainty. Festival organizers can trust such a decisionmaking tool in order to optimize their planning, operational resources and costs. Here, the load demand was considered perfectly known, based on the fact that most loads are known beforehand (e.g., lighting needs). Nevertheless, uncertainty introduced by an unusually large number of visitors could be considered in future work. 


\section{ACKNOWLEDGEMENT}

This research has received funding through the ERA-Net Smart Grids Plus project Grid- Friends, with support from the European Unions Horizon 2020 research and innovation programme. This work is part of the Veni research programme with project number 639.021.751, which is financed by the Netherlands Organisation for Scientific Research (NWO). The Authors would like to kindly thank Watt-now [10] for providing anonymized festival data.

\section{REFERENCES}

[1] R. Doherty and M. O'Malley, "A new approach to quantify reserve demand in systems with significant installed wind capacity," IEEE Transactions on Power Systems, vol. 20, no. 2, pp. 587-595, May 2005.

[2] S. H. Madaeni and R. Sioshansi, "The impacts of stochastic programming and demand response on wind integration," Energy Systems, vol. 4, no. 2, pp. 109-124, 2013. [Online]. Available: http://dx.doi.org/10.1007/s12667-012-0068-7

[3] T. Niknam, R. Azizipanah-Abarghooee, and M. R. Narimani, "An efficient scenario-based stochastic programming framework for multi-objective optimal micro-grid operation," Applied
Energy, vol. 99, pp. 455 - 470, 2012. [Online]. Available: http://www.sciencedirect.com/science/article/pii/S0306261912003030

[4] G. Cardoso, M. Stadler, A. Siddiqui, C. Marnay, N. DeForest, A. Barbosa-Pvoa, and P. Ferro, "Microgrid reliability modeling and battery scheduling using stochastic linear programming," Electric Power Systems Research, vol. 103, pp. 61 - 69, 2013. [Online]. Available: http://www.sciencedirect.com/science/article/pii/S0378779613001235

[5] Z. Chen, L. Wu, and Y. Fu, "Real-time price-based demand response management for residential appliances via stochastic optimization and robust optimization," IEEE Transactions on Smart Grid, vol. 3, no. 4, pp. 1822-1831, Dec 2012.

[6] N. G. Paterakis, I. N. Pappi, J. P. S. Catalo, and O. Erdinc, "Optimal operation of smart houses by a real-time rolling horizon algorithm," in 2016 IEEE Power and Energy Society General Meeting (PESGM), July 2016, pp. 1-5.

[7] J. R. Birge and F. Louveaux, Introduction to Stochastic Programming, 2nd ed. Springer Publishing Company, Incorporated, 2011.

[8] "ELIA solar power generation data," http://www.elia.be/en/griddata/power-generation/Solar-power-generation-data/Graph, accessed: $1 / 9 / 2017$.

[9] "ENTSO-E Transparency Platform," https://transparency.entsoe.eu/, accessed: $1 / 9 / 2017$

[10] "Watt-Now: Organiseer schonere festivals," https://www.watt-now.com/, accessed: 1/9/2017. 